\title{
Assessment of the Quality of Malaria Rapid Diagnostic Test, Adama District, East Shewa Zone, Ethiopia
}

\author{
Merga Gonfa $^{1}$, Daba Mulleta $^{2}$, Wakgari Deresa ${ }^{3}$, Bizuayehu Gurmesa ${ }^{1}$ \\ ${ }^{1}$ Department of Medical Laboratory Science, College of Health Science, Arsi University, Asella, Ethiopia \\ ${ }^{2}$ Oromia Public Health Research Capacity Building and Quality Assurance Laboratory, Adama, Ethiopia \\ ${ }^{3}$ School of Public Health, College of Health Science, Addis Ababa University, Addis Ababa, Ethiopia
}

Email address:

sesimego@gmail.com (M. Gonfa)

\section{To cite this article:}

Merga Gonfa, Daba Mulleta, Wakgari Deresa, Bizuayehu Gurmesa. Assessment of the Quality of Malaria Rapid Diagnostic Test, Adama District, East Shewa Zone, Ethiopia. International Journal of Clinical and Experimental Medical Sciences. Vol. 6, No. 6, 2020 , pp. 109-118. doi: $10.11648 /$ j.ijcems.20200606.11

Received: September 21, 2020; Accepted: October 6, 2020; Published: November 23, 2020

\begin{abstract}
Background: In Ethiopia, smear microscopy is the gold standard for malaria diagnosis however; it is not available in health post. Smear microscopy is time consuming, requires trained personnel and needs careful preparation and application of reagents to ensure quality results. Objective: This study was aimed at testing the diagnostic performance of SD BIOLINE malaria rapid diagnostic test (RDT) with reference to smear microscopy for the diagnosis of falciparum and vivax malaria in Ethiopia. Methods: Blood samples were collected from 402 patients suspected to have malaria in four health facilities in the late minor malaria transmission season from June18-30, 2014. The samples were examined immediately by smear microscopy and the RDT (SD BIOLINE Malaria HRP2/pLDH POCT Test kit). Statistical analysis was performed using Epi-info version 7 and the two-way contingency table analysis. Results: The overall parasite positivity using smear microscopy was 163 (45.6\%): 136 (33.8\%) for P. falciparum, 25 (6.2\%) for P. vivax and two (0.5\%) for mixed infections. Using the SD BIOLINE RDT, the overall parasite positivity was 176 (43.7\%): 149 (37\%) for P. falciparum, 26 $(6.5 \%)$ for P. vivax and $1(0.2 \%)$ for mixed infections. The overall sensitivity and specificity of SD BIOLINE RDT was found to be $98.2 \%(97.5-99.9 \%, 95 \% \mathrm{CI})$ and $95.2 \%(93.9-97.7 \%, 95 \% \mathrm{CI})$, respectively. The sensitivity and specificity of SD BIOLINE RDT was found to be 98.2\% (94-99.1\%, 95\% CI) and 95.4\% (93.9-97.1\%, 95\% CI), respectively. The positive predictive value (PPV) and the negative predictive value (NPV) were found to be 93.6\% (88.5-96.1\%, 95\% CI) and 98.7\% (95.3-99.8\%, 95\% CI), respectively. There was an excellent agreement between the smear microscopy and SD BIOLINE RDT with a Kappa value of 0.965 (0.896-0.988, 95\% CI). Conclusion: The SD BIOLINE RDT test showed good sensitivity and specificity with an excellent agreement to the reference smear microscopy. The RDT could therefore be used in place of smear microscopy, at health post where the microscope not applicable.
\end{abstract}

Keywords: Malaria, Rapid Diagnostic Test, East Shoa Zone, Ethiopia

\section{Introduction}

\subsection{Background}

Malaria continues to be one of the main public health problems in the world, especially in a majority of African countries. (WHO) estimates 1,272,000 deaths have occurred globally with Africa bearing the majority of deaths: 1,136,000 (89.3\%), South-East Asia: 65,000 (5.1\%), Americans: 1000 (less than 1\%), Western Pacific: 11,000 (less than 1\%), and Eastern Mediterranean: 59,000 (4.6\%).
To overcome malaria challenges, there is a need for concerted efforts in the management of malaria cases using accessible and rapid diagnostic tools by health services, private sector, and local communities [1].

Malaria is a major public health problem in Ethiopia and has been consistently reported as one of the three leading causes of morbidity and mortality. Plasmodium falciparum and Plasmodium vivax are the two dominant parasite species that cause malaria in Ethiopia, with frequencies of about $60 \%$ and $40 \%$, respectively [2]. This proportion varies by location and season. Plasmodium falciparum is the dominant parasite 
species in malaria epidemic situations, and this species causes severe and complicated manifestations and almost all malaria deaths. Plasmodium falciparum has a remarkable biological diversity including an ability to develop resistance rapidly to a number of anti-malarial drugs, creating a major challenge in providing patients with this infection with effective malaria chemotherapy $[1,2]$.

In Ethiopia, malaria is one of the most important public health problems, with more than three-quarters of the landmass (altitude $<2000 \mathrm{~m}$ ) of the country either malarious or potentially malarious. An estimated $68 \%$ (>50 million people) of the total population resides in areas at risk of malaria infection. Annually, half a million microscopically confirmed cases of malaria are reported to the Federal Ministry of Health (FMOH) from basic health services.

Despite its high public health importance, the validity of the RDT test against the gold standard microscopic examination is not well understood in the country. However, the actual number of malaria cases in the country is estimated to be more than 5 million each year. According to the 2007/2008 report of the $\mathrm{FMOH}$, malaria was the leading cause of outpatient visit accounting for $12 \%$ of cases and the second cause of (10\%) admission next only to admissions for delivery [3].

The national government of Ethiopia is taking aggressive control measures like, case treatment, distribution of insecticide treated nets (ITNs), indoor residual spray and health education at grass root level by health extension workers. Malaria presents a diagnostic challenge to laboratories in most countries including Ethiopia. The urgency and importance of obtaining test results quickly from the examination of blood samples from patients with suspected acute malaria render some of the more sensitive methods for malaria diagnosis impractical for routine laboratory use. In our country both microscopic and rapid diagnostic test (RDT) malaria methods are used. The validity of RDT test against the golden standard microscopic examination is not regularly reviewed as one it verified at national and not well understands in Oromia region [3].

The national data indicates that when malaria is reported "clinical + confirmed" it is the number one cause of morbidity and mortality; when it reported "confirmed" only, it is the number five cause of morbidity and mortality. In Ethiopia, P. falciparum constitutes the larger proportion of cases detected by microscopy $(77 \%)$ in areas $<2,000 \mathrm{~m}$. In Oromia, however, $P$. vivax was the main etiologic agent of cases confirmed by microscopy, with $60 \%$ of slide-positive cases. Oromia also had the lowest malaria prevalence $(0.5 \%)$ compared to other Ethiopian regions. Nationally, males were found to be more affected than females (1.6\% versus $1.0 \%)$ respectively. The highest proportion of RDT-positive malaria cases was found in age group 10-19 (5.5\%) and the lowest in age group 70-79 (1.1\%) [4].

Ninety percent of the districts in East Shewa Zone are estimated to be malarious. This is due to existence of natural and manmade lakes (Ziway and Koka), sugar factories (Wanji and Metehara) and expansions of newly constructed water and irrigation project in Fantale woreda. Even though malaria control measures have been under way in the zone, malaria weekly integrated disease surveillance report (IDSR) and monthly health management information system (HMIS) reports indicated that East Shewa is one of the leading zones reporting malaria cases in the region in the last 5years.

\subsection{Statement of the Problem}

In Ethiopia, annually, half a million microscopically confirmed cases of malaria are reported to the FMOH from basic health posts. However, the actual number of malaria cases in the country is estimated to be more than 5 million each year. According to the 2012/2013 report of the FMOH, malaria was the third leading cause of outpatient visits accounting for nine percent of cases and the second cause of $(5 \%)$ admission next only to admissions for delivery $[3,5]$.

In the prevention and control of malaria, prompt and accurate diagnosis is the key to effective disease management [5] however, in Ethiopia, clinical diagnosis and empirical treatment has been the mainstay of malaria management in areas where laboratory facilities are not available. Due to the non-specific nature of signs and symptoms of malaria, clinical diagnosis is unreliable $[3,5,6]$. In many countries malaria is still being diagnosed clinically, an unreliable method leading to over-diagnosis and over-treatment [7]. Light microscopy (LM) remains preferred and standard for laboratory diagnosis of malaria although it is not accessible and affordable in most peripheral health facilities in the country. Moreover, microscopy is time consuming, requires trained personnel and needs careful preparation and application of reagents to ensure quality results $[6,8]$. Confirmatory diagnosis before treatment initiation recently regained attention, partly influenced by the spread of drug resistance and thus the requirement of more expensive drugs unaffordable to resource-poor countries [5]. For a better and sustainable control, malaria diagnosis requires a more rapid, easy, sensitive and specific method.

\subsection{Literature Review}

The burden of malaria has been increasing due to a combination of large population movements, increasing large scale epidemics, mixed infections of $P$. vivaxand, $P$. falciparum, increasing parasite resistance to malaria drugs, vector resistance to insecticides, low coverage of malaria prevention services and general poverty. Outpatient consultations, inpatient admissions and all in-patient deaths have risen by $21-23 \%$ over the last five years. Ethiopian adults, unlike their counterparts in more endemic areas, have relatively little protective immunity and are also vulnerable to malaria epidemics, which traditionally occur every five to eight years, are a hallmark of malaria in Ethiopia. The epidemic of 1950 is estimated to have caused 3 million cases and resulted in 150,000 deaths. Unstable and largely unpredictable malaria epidemiology makes surveillance, information management and logistics for vector control and pharmaceuticals of paramount importance. Plasmodium vivax 
and Plasmodium falciparum comprise $40 \%$ and $60 \%$ of malaria infections respectively [8].

Malaria presents a diagnostic challenge to laboratories in most countries. Endemic malaria, population movements, and travelers all contribute to presenting the laboratory with diagnostic problems for which it may have little expertise available. Drug resistance and genetic variation has altered many accepted morphological appearances of malaria species, and new technology has given an opportunity to review available procedures. Concurrently the World Health Organization has opened a dialogue with scientists, clinicians, and manufacturers on the realistic possibilities for developing accurate, sensitive, and cost-effective rapid diagnostic tests for malaria capable of detecting 100 parasites / $\mu 1$ from all species and with a semi-quantitative measurement for monitoring successful drug treatment. New technology has to be compared with an accepted gold standard that makes comparisons of sensitivity and specificity between different methods. The majority of malaria is found in countries where cost-effectiveness is an important factor and ease of performance and training is a major consideration. Most new technology for malaria diagnosis incorporates immune chromatographic capture procedures, with conjugated monoclonal antibodies providing the indicator of infection. Preferred targeted antigens are those which are abundant in all asexual and sexual stages of the parasite and are currently centered on detection of HRP-2 from Plasmodium falciparum and parasite-specific lactate dehydrogenase or Plasmodium aldolase from the parasite glycolytic pathway found in all species. Clinical studies allow effective comparisons between different formats, and the reality of non-microscopic diagnoses of malaria is considered [9].

The current gold standard for laboratory confirmation of diagnosis of malaria is a peripheral blood smear, examined microscopically. However trained staff and quality equipment and supervision are scarce within populations requiring such diagnosis. Alternative methods of diagnosis are available, the simplest of which are RDT. These are antigen detection tests which are simple to use and to interpret, and also use peripheral blood. Currently the RDTs which have been most developed detect an antigen called histidine reach protein II (HRP2) produced by P.falciparum trophozoites and young gametocytes [10].

Malaria RDTs are used increasingly for diagnosis of malaria, particularly in remote tropical areas where good microscopybased diagnosis is impractical. RDTs must therefore be robust, simple and safe to use, and reliably demonstrate when malaria parasitaemia is, and is not, present $[4,11]$.

The overall parasite positivity using light microscopy was (40.9\%): $15.7 \%$ for P. falciparum, $22.8 \%$ for P. vivax and $2.4 \%$ for mixed infections. Using the Care Start ${ }^{\mathrm{TM}} \mathrm{RDT}$, the overall parasite positivity was (39.4\%): (19.7\%) for P. falciparum, (9.5\%) for P. vivax and (10.2\%) for mixed infections [12].

Care Start $^{\mathrm{TM}}$ RDT showed low sensitivity in over allPlasmodium (90.8\%) and P. falciparum (87.5\%) in household survey; and in P. vivax $(92.8 \%)$ in health facility surveys. Similarly, low specificity observed in overall
Plasmodium (82.7\%), and P. falciparum (92.8\%) in health facility surveys, and in P.vivax (87.5\%) in household surveys. Moreover, lowest PPV was determined in overall Plasmodium (64.3\%) and P.falciparum (77.2\%) in health facility; and overall Plasmodium (76.7\%) and P. falciparum $(87.5 \%)$ in household surveys. Negative predictive value of the test was good in both overall Plasmodium and P. falciparum. However, lowest NPV was found in P. vivax in both health facility (77.2\%) and household (87.5\%) surveys [13].

\subsection{Purpose of the Study}

In Ethiopia, malaria is a common public health problem and is among the three leading causes of morbidity and mortality. Malaria presents a diagnostic challenge to laboratories in most countries including Ethiopia. The urgency and importance of obtaining test results quickly from the examination of blood samples from patients with suspected acute malaria render some of the more sensitive methods for malaria diagnosis. RDTs are used increasingly for diagnosis of malaria, particularly in remote tropical areas where microscopy-based diagnosis is impractical. RDTs must therefore be robust, simple and safe to use, and reliably demonstrate when malaria parasitaemia is, and is not, present. In Ethiopia both Microscopic and RDT malaria diagnostic test methods are used. The validity of RDT tests against the gold standard microscopic examination is not well understood in the country. This study was conducted to evaluate the sensitivity and specificity of SD BIOLINE malaria Antigen P.f/ P.v POCT" RDT test in reference to the conventional smear microscopy in north-west Ethiopia.

\section{Objectives}

\subsection{General Objective}

The purpose of this study is to determine the quality (sensitivity, specificity, PPV, NPV) of rapid diagnostic test in comparison to gold standard microscopy.

\subsection{Specific Objectives}

1) To measure the sensitivity of rapid test compared to microscopy.

2) To assess specificity of rapid test when compared to microscopy.

3) To describe the reliability of the rapid diagnostic malaria laboratory test.

\section{Methods and Materials}

\subsection{Study Area}

The study was conducted in Adama district of East Shewa zone of Oromia region. It is one of the 18 zones found in Oromia regional state government. East Shewa zone is found in the central-eastern part of county and the capital, Adama town, is located along the high way to Harar, $100 \mathrm{Km}$ from 
Addis Ababa. The total population of the zone (including the town administrations) is $1,584,064$ according to the regional health bureau annual planning document. Adama district surrounding Adama town is one of the districts in East Shewa Zone having a total population of 180,710 and administratively the district was divided into 42 (37 rural and 5 urban) kebeles. The district provides health service with one district hospital, seven health centers and 36 health posts. The primary health services coverage was $100 \%$ in 2013 .

\subsection{Study Design and Study Period}

A health facility based cross-sectional diagnostic evaluation study was conducted. A structured questionnaire was used to collect information on demographic and socioeconomic characteristics of the patients. The information was collected by selected and trained health professionals (nurse and laboratory technicians) working in randomly selected health facilities (hospital and Health centers). Patients with malaria suspected fever cases that present to selected health facilities were examined by using one step malaria antigen point of care test "SD BIOLINE Malaria Antigen P.f/P.v POCT" RDT and diagnosed by smear microscopy. Patient's positive for malaria was treated as usual with the recommended regimen according to national malaria treatment guide line.

Data was collected from randomly selected health facilities (Awash Melkasa, Shewa Alem Tena) and Wonji Hospital) from June $18^{\text {th }}-30^{\text {th }}, 2014$.

\subsection{Source, Study Population and Sample Size Calculation}

The total population /residents of district and study units were all patients fever malaria suspected cases visited to selected health centers and hospitals from June $18^{\text {th }}-30^{\text {th }}$, 2014.

Random sampling method was used and selects two health centers (Awash Melkasa and Shewa-AlemTena) and Wonji Hospital for data collection in from the district. Convenient sampling technique was used for selecting the study participants considering $95 \% \mathrm{CI}, 20 \% \beta$ - error allowing $10 \%$ dropout. Finally a total of 402 fever malaria suspected cases were included in the study sample. Both thick and thin blood smears were performed on a single slide by experienced and trained laboratory technologist /technicians. Peripheral blood examination for malaria parasites were collected at the same time for blood smear slides and "SD BIOLINE malaria Antigen P.f/ P.v POCT" RDT test for each study participant who volunteer and involved in the study. Respondents were asked about the signs and symptoms of malaria and medications taken prior the investigation by clinician outpatient diagnosis room.

\subsection{Study Variables}

\subsubsection{Dependent Variable}

Sensitivity, specificity, predictive value positive and predictive value negative.

\subsubsection{Independent Variables}

Patient characteristics sex, age and educational status.

\subsection{Inclusion and Exclusion Criteria}

\subsubsection{Inclusion}

All malaria suspected febrile cases visited selected health facilities from June $18^{\text {th }}-30^{\text {th }}, 2014$ and volunteer to participate in the study were included.

\subsubsection{Exclusion}

Patients who already took malaria treatment two weeks prior and had come back again for treatment were excluded from the study.

\subsection{Data Collection and Data Collection Tools}

A structured questionnaire was developed; sociodemographic characteristics and clinical data of the participants were collected using a structured questionnaire. We collected information from parents (i.e. father, mothers/caregiver) about the child for those under 14 years age. The questionnaire was per-tested before the main data collection was started finally the pilot data was included in the analysis.

1) Nurses and laboratory technician working in the selected health centers and hospital were selected and trained on data collection procedure for one day before main data collection.

2) Data collectors were interviewed study participants who full fill criteria and willing to participate on demographic condition during the study period using a structured questionnaire.

Specimen collection and processing

Finger-prick samples were collected and placed in a grease-free, clean, glass slide. The same finger-prick blood sample was used to carry out the RDT in parallel, following manufacturer's instructions. In a single slide, both thick and thin films were prepared. The thin films were fixed in methanol after air-drying; the slides were stained in $10 \%$ Giemsa solution for $15 \mathrm{~min}$.

\subsubsection{Material and Supply Used for Data Collection}

1) Olympus microscope

2) Immersion oil with refractive index of $\geq 1.5$

3) SD BIOLINE malaria Ag P.f/P.v POCT RDT kit

4) Giemsa stock solution

5) Buffer tablets

6) Slide racks

7) Methanol

8) Frosted slides

9) Mineral water (PH 7.2)

10)Slide box

\subsubsection{Laboratory Procedure}

SD BIOLINE malaria antigen P.f/P.v POCT

1. Allow all kit component to room temperature before testing

2. Remove the test device from the foil pouch, place it on 
the flat, dry surface

3. Clean the fingertip and prick finger with lancet

4. With a5 $\mu$ l capillary tube provided collect whole blood specimen to black line and then transfers down whole blood in the round sample well. Or with a $5 \mu 1$ disposable inverted cap circular end of the loop in to the blood specimen and carefully place the circular end of the loop in the round sample well. Or with a $5 \mu 1$ disposable inverted cup provided. Dip the circular end of inverted cup in to the blood specimen and carefully place the circular end of the inverted cup in the round sample well.

5. Twist and pull tab to open assay diluents as figure

6. Dispense all of the assay diluents from the diluents tube in to the square well of test device

7. Waite minimum of 15 minutes (up to $30^{\prime}$ ) and read the result.

NB: - Not read test results after 30 minutes.

Smear microscopy

1. Clean the figure tip with alcohol then wait until dry

2. Puncture the figure tip with lancet quickly

3. Wipe first drop of blood away with a clean, dry gauze

4. Apply small drop of blood on single slide for both $6 \mu 1$ for thick and $4 \mu \mathrm{l}$ for thin film

5. Use dry cotton and inform patient press until blood stop

6. Making the thin smear (Angle controls the spread; 3045 degree angle preferred

7. Labeling project ID, pt ID, date \& time collection on slide

8. Allow the blood film to dry on a flat, firm surface



Figure 1. Drying Thick and Thin blood film.

9. Fixing with methanol

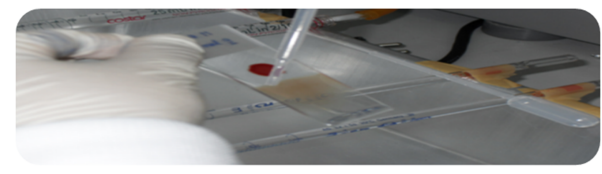

Figure 2. Fixing thin blood film with methanol alcohol.

10. Drying vertically

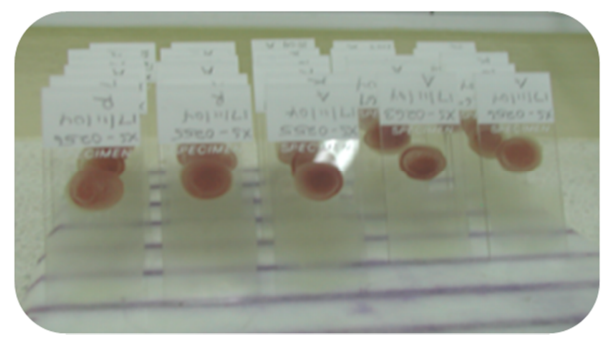

Figure 3. Drying blood filmvertically after fixation with methanol.
Staining: Thick and thin blood smears

1. Place the slides flat on a drying/ staining rack



Figure 4. Thik and thin blood smears on staining rack.

2. Flood the slides with stain $10 \%$ for 15 minutes

3. Gently wash the slides to keep the thick films in place; individually wash to avoid cross contamination

4. Drying

5. Thin and thick films were read using Olympus KX-21 binocular microscope at Adama malaria center by an experienced laboratory technician and the result was considered negative if no parasites were seen after examination of 200 fields at $1,000 x$ magnification.

\subsection{Operational Definitions}

RDT was interpreted in the following manner;

1) One line at the control position: Negative

2) One line at control position plus one line at the test position: Positive

3) One line at the test position and no line at control position or no line present: Invalid

\subsection{Data Processing and Analysis}

Data was entered in to Epi-Info software, cleaned, processed and analyzed. We used two by two tables to evaluate the test performance (i.e. specificity, sensitivity, PPV and NPV). We used frequency tables and graphs for descriptive statistics such as socio demographic data.

\subsection{Data Quality}

The laboratory technician/technologist prepared blood smear and examined RDT and the clinician who collected information on demographic characteristics were recruited from the selected Awash Melkasa, Shewa-AlemTena health centers and Wonji Hospital. They were trained for one day to become familiar with the data collection tool. On the same day afternoon the tool was tested in the same facilities. Data collection tools were revised and adjustment was made based on findings before initiated actual data collection.

The questionnaire was prepared in English and translated to local language "Afan Oromoo" for field work purposes, then back translated to English by different individuals in order to check for language consistency.

SD BIOLINE malaria Ag P.f/P.v POCT RDT kit storage quality was assured before implementing to the actual data collection using DTS panel prepared by CDC for the pilot project. Room temperature for storage of RDT kit was daily monitored at health facility level during data collection. We 
were recruited highly experienced laboratory technician/technologists from Adama malaria center.

We were verified quality of microscope and immersion oil by known blood smear prepared and validated Oromia public health research, capacity building and quality assurance laboratory. All blood films were re-read by an experienced microscopist at Oromia Public health Research and capacity building and quality assurance laboratory that was also blinded to initial microscopy and RDT results. In cases where the results were discordant, a third expert reader was used. The results of the third expert reader were considered final.

We used second and third readers were from Oromia Public health Research and capacity building and quality assurance laboratory. The laboratory is labeled as two stars by African Society of Laboratory Medicine (ASLM) which is on the final stages of accreditation by the WHO-AFRO. The laboratory is involved in external quality assurance (EQA) at International by digital Proficiency testing from one world accuracy and EPHI.

\subsection{Ethical Consideration}

Ethical clearance was secured from the Research Ethics Committee of Adama Hospital Medical College and we got supportive letter from Oromia Regional Health Bureau. We were presented supportive letter to East Shewa Zone Health Department (ZHD) and Adama District Health Office (DHO) and got permission to conduct the study. We were secured written consent form the study subjects who agreed to participate on the study. Confidentiality and anonymity of the study participants and study findings were maintained. All malaria confirmed cases were treated immediately by the standard treatment protocol at the health facilities level according to national malaria treatment guide line.

\section{Result}

\subsection{Socio-demographic Characteristics}

A total 402 febrile suspected malaria cases were examined by RDT in three health facilities of Adama district. Majority of the suspected cases were Oromo ethnic and orthodox was the predominant religion in the area accounting for $60.4 \%$. About $35.1 \%$ of suspected cases were students followed by house wife and farmers $17.9 \%$ and $12.4 \%$ respectively (Table 1 ).

Table 1. Socio-demographic characteristics of suspected malaria cases in Adama District, East Shewa Zone, 2014.

\begin{tabular}{|c|c|c|}
\hline Characteristics & Frequency & Percent \\
\hline \multicolumn{3}{|l|}{ Sex } \\
\hline Female & 174 & 43.3 \\
\hline Male & 228 & 56.7 \\
\hline \multicolumn{3}{|l|}{ Age group } \\
\hline $0-4$ & 43 & 10.7 \\
\hline $5-9$ & 47 & 11.7 \\
\hline $10-14$ & 49 & 12.2 \\
\hline $15-44$ & 223 & 55.5 \\
\hline $45+$ & 40 & 10.0 \\
\hline \multicolumn{3}{|l|}{ Religious } \\
\hline Orthodox & 243 & 60.4 \\
\hline Portestant & 112 & 27.9 \\
\hline Muslim & 33 & 8.3 \\
\hline Wakefata & 11 & 2.7 \\
\hline Catholic & 3 & 0.7 \\
\hline \multicolumn{3}{|l|}{ Occupation } \\
\hline Students & 141 & 35.1 \\
\hline House wife & 72 & 17.9 \\
\hline Farmer & 51 & 12.7 \\
\hline Daily labour & 50 & 12.4 \\
\hline Under age & 38 & 9.5 \\
\hline Government employee & 27 & 6.7 \\
\hline Merchants & 8 & 2.0 \\
\hline Driver & 3 & 0.7 \\
\hline Others & 12 & 3.0 \\
\hline Total & 402 & 100 \\
\hline
\end{tabular}

Of the total suspected malaria cases $228(56.7 \%)$ and 174 $(43.3 \%)$ were males and females respectively in Adama District from June $18^{\text {th }}-30^{\text {th }}, 2014$. The median age of malaria suspected cases were 20 years with range of 1 to 82 years were registered. About $34.6 \%$ of the study participants were below 14 year age and with no gender differences (Table 1).

Of the total study participants of suspected malaria cases tested by RDT 170 (42.3\%) were positive, in Adama district $64.1 .9 \%$ were identified among male and the proportion of Plasmodium falciparum specie among male cases was $63.4 \% .58 .8 \%$ of the confirmed malaria cases were identified among 15-44 age group followed by 10-14 and 5-9 aged (13.5\%). The overall proportion of Plasmodium faciparum species was 50.5\% among 15-44 age groups (Table 2 ).

Table 2. Distribution of study participant by sex, age group and Plasmodium species in Adama district, June, 2014.

\begin{tabular}{|c|c|c|c|c|c|}
\hline \multirow{2}{*}{ Characteristics } & \multirow{2}{*}{ RDT tested № (\%) } & \multirow{2}{*}{$\begin{array}{l}\text { Total Positive } \\
\text { № (\%) }\end{array}$} & \multicolumn{3}{|c|}{ Plasmodium species } \\
\hline & & & P.f № (\%) & P.v № (\%) & Mixed № (\%) \\
\hline \multicolumn{6}{|l|}{ Sex } \\
\hline Male & $228(56.7)$ & $109(64.1)$ & $90(63.4)$ & $18(66.7)$ & $1(100)$ \\
\hline Female & $174(43.3)$ & $61(35.9)$ & $52(36.6)$ & $9(33.3)$ & $0(0)$ \\
\hline Total & $402(100)$ & $170(100)$ & $142(100)$ & $27(100)$ & $1(100)$ \\
\hline \multicolumn{6}{|l|}{ Age group } \\
\hline $0-4$ & $43(10.7)$ & $15(8.8)$ & $11(7.7)$ & $4(15.4)$ & $0(0)$ \\
\hline $5-9$ & $47(11.7)$ & $23(13.5)$ & $21(14.8)$ & $2(7.7)$ & $0(0)$ \\
\hline $10-14$ & $49(12.2)$ & $23(13.5)$ & $16(11.3)$ & $7(26.9)$ & $0(0)$ \\
\hline $15-44$ & $223(55.5)$ & $100(58.8)$ & $86(60.6)$ & $13(50)$ & $1(0)$ \\
\hline $45+$ & $40(9.9)$ & $9(5.3)$ & $8(5.6)$ & $1(3.9)$ & $1(100)$ \\
\hline Total & $402(100)$ & $170(100)$ & $142(100)$ & $27(100)$ & $1(100)$ \\
\hline
\end{tabular}


Of the total 402 study participants $73.4 \%$ and $26.6 \%$ were from urban and rural respectively. Most of the P.falciparum malaria cases were reported from urban $56.3 \%$ (Table 3 ).

Table 3. Residence of study participants in Adama district, East Shewa zone from June, 2014.

\begin{tabular}{|c|c|c|c|c|c|}
\hline \multirow[b]{2}{*}{ Residence } & \multirow{2}{*}{ Total RDT tested № (\%) } & \multirow{2}{*}{$\begin{array}{l}\text { Total Positive№ } \\
\text { (\%) }\end{array}$} & \multicolumn{3}{|l|}{ Plasmodium Species } \\
\hline & & & P. falciparum № (\%) & P. vivax № (\%) & Mixed № (\%) \\
\hline Urban & $295(73.4)$ & $97(57.1)$ & $80(56.3)$ & $16(59.3)$ & $0(0)$ \\
\hline Rural & $107(26.6)$ & $73(42.9)$ & $62(43.7)$ & $11(40.7)$ & $1(100)$ \\
\hline Total & $402(100)$ & $170(100)$ & $142(100)$ & $27(100)$ & $1(100)$ \\
\hline
\end{tabular}

The highest proportion of total confirmed malaria cases and plasmodium falciparum were identified in urban $57.1 \%$ and $56.3 \%$ respectively (Figure 5).

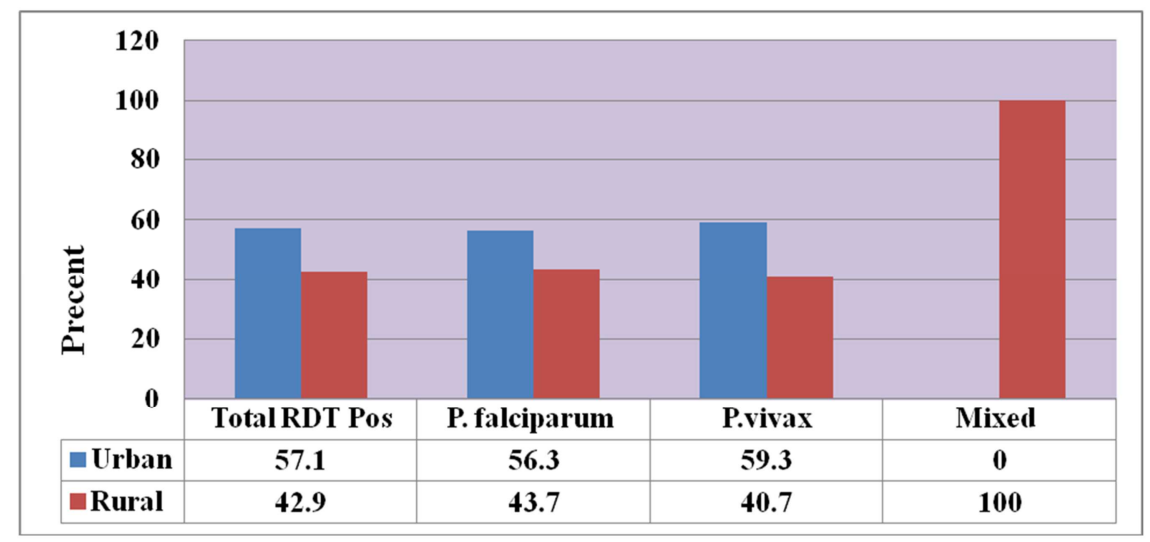

Figure 5. Proportions of total and Plasmodium falciparum malaria cases by residence of the study participants in Adama district, East Shewa zone, June, 2014.

Majority of data were collected from Awash Melkasa health center and most RDT positive cases were identified in Awash Melkasa health center $(85.9 \%)$ About $69.2 \%$ of the study participants had ITN and $68.4 \%$ of them were positive for P.falcipaurm (Table 4).

Table 4. Distribution of study participants by health facilities in Adama district, East Shewa zone from June, 2014.

\begin{tabular}{llllll}
\hline \multirow{2}{*}{ Health Facilities } & RDT tested & Total Positive & \multicolumn{2}{l}{ Plasmodium Species } & \\
\cline { 3 - 5 } & № (\%) & № (\%) & P. falciparum № (\%) & P. vivax № (\%) & Mixed № (\%) \\
\hline A/Melkasa HC & $244(60.7)$ & $146(85.9)$ & $123(86.6)$ & $22(81.5)$ & $1(0)$ \\
S/AlemTena HC & $72(17.9)$ & $13(7.6)$ & $12(8.5)$ & $1(3.7)$ & $1(100)$ \\
Wonji Hosp & $86(21.4)$ & $11(6.5)$ & $7(4.9)$ & $4(14.8)$ & $0(0)$ \\
Total & $402(100)$ & $170(100)$ & $142(100)$ & $27(100)$ & $58(42.2)$ \\
\hline
\end{tabular}

Fever was the most commonly reported presenting symptom by the participants $(88.3 \%)$ while headache (79.8\%), sweating/chills/rigors (67.1\%), fatigue (62.7\%), and vomiting $(35 \%)$ were other common presenting features.

\subsection{Method Performance}

All of the study participates were examined by RDT at selected health facilities and smear microscopy at Adama malaria center by level-I malaria experts. The overall parasite positivity using smear microscopy was 163 (40.5\%): 136 (33.8\%) for P. falciparum, $25(6.2 \%)$ for P. vivax and two $(0.5 \%)$ for mixed infections. However, SD BIOLINE RDT positivity was $170(42.3 \%)$ : $142(35.3 \%)$ for P. falciparum, $27(6.7 \%)$ for P. vivax and $1(0.2 \%)$ for mixed infections (table 2). Difference in detection of malaria parasites using either the smear microscope or the RDT was insignificant. On other hand $3(0.7 \%)$ malaria suspected fever cases were negative by RDT at health facilities but positive by smear microscopy at malaria center for any plasmodium species (Table 6).

Table 5. Fever among the study participants in Adama District, East Shewa Zone June, 2014.

\begin{tabular}{llllll}
\hline \multirow{2}{*}{ Fever } & \multicolumn{2}{l}{ RDT Result } & \multicolumn{2}{l}{ Microscopic Result } & \multirow{2}{*}{ Total } \\
\cline { 2 - 5 } & Positive & Negative & Positive & Negative & \\
\hline Yes & 173 & 182 & 161 & 194 & 355 \\
No & 3 & 44 & 2 & 45 & 47 \\
Total & 176 & 226 & 163 & 239 & 402 \\
\hline
\end{tabular}

Table 6. Performance evaluation of RDT compared to microscopic malaria diagnostic method, in Adama District, East Shewa Zone June, 2014.

\begin{tabular}{llll}
\hline RDT Malaria & \multicolumn{3}{l}{ Microscopic Examination } \\
\cline { 2 - 4 } diagnostic method & Positive & Negative & Total \\
\hline Positive & 160 & 10 & 170 \\
Negative & 3 & 229 & 232 \\
Total & 163 & 239 & 402 \\
\hline
\end{tabular}


1) Sensitivity of the test

$$
\text { Sensitivity }(\mathrm{Se})=160 / 163 \times 100=98.2 \%
$$

2) Specificity of the test

$$
\text { Specificity }(\mathrm{SP})=228 / 239 \text { X100 }=95.4 \%
$$

3) Positive predictive value (PPV) of the test

Positive predictive value $(\mathrm{PPV})=160 / 170 \mathrm{X} 100=94.1 \%$

4) Negative predictive value (NPV) of the test

Negative predictive value $(\mathrm{NPV})=229 / 232 \mathrm{X} 100=98.7 \%$

5) Kappa, K

$$
\mathrm{K}=\frac{\left(p_{o}-p_{e}\right)}{\left(1-P_{e}\right)}=\frac{0.96766-0.51457}{1-0.51457}=0.9334
$$

Taking the smear microscope as a standard test for malaria, the sensitivity and specificity of SD BIOLINE RDT was found to be $98.2 \%(94-99.1 \%, 95 \% \mathrm{CI})$ and $95.4 \%(93.9-97.1 \%, 95 \%$ $\mathrm{CI}$ ), respectively. The positive predictive value (PPV) and the negative predictive value (NPV) were found to be $94.1 \%(88.5-$ $96.1 \%, 95 \% \mathrm{CI})$ and $98.7 \%(95.3-99.8 \%, 95 \% \mathrm{CI})$, respectively. There was an excellent agreement between the smear microscopy and SD BIOLINE RDT with a Kappa value of $0.9334(0.896-0.988,95 \%$ CI) (table 6).

\section{Discussion}

Majority of the malaria diagnostic methods help to identified malaria cases and to early intervention and minimized the delayed of the treatment. World Health Organization recommends that parasitolgic malaria conformation for diagnosis of malaria cases. Currently SD BIOLINE malaria Ag P.f/P.v laboratory diagnostic methods performed for the fever malaria suspected cases at community level by health professionals other than laboratory personnel to early identification and initiate treatment.

The present study revealed a high sensitivity and specificity of the SD BIOLINE malaria Ag P.f/P.v laboratory. The high sensitivity of the RDT in this study was in line with other study from south-west and North Ethiopia. Overall, the SD BIOLINE malaria Ag P.f/P.v RDT showed good sensitivity when compared to the smear microscopy. In set ups where health personnel rely on their clinical judgment, using RDT for the diagnosis of malaria can be helpful for early institution of treatment.

This study had also tried to evaluate the performance of the SD BIOLINE malaria Ag P.f/P.v in detecting different species of malaria parasite. The finding in the current study was higher than that reported by Ashton et al from Oromia Regional State of Ethiopia [14]. The sensitivity of the RDT in the current study for P. falciparum or mixed infection was also higher than that by Maltha et al (78.5\%) [15]. However, the sensitivity in this study was found to be lower than reports from south-west Ethiopia [16] south Ethiopia [7] and Madagascar [18]. These differences could be due to observer variation, difference with malaria species circulating at different localities or host factors [19].

The specificity of the SD BIOLINE malaria Ag P.f/P.v in the present study for P. falciparum or mixed infections was higher than the reports from Oromia Regional State in Ethiopia and Madagascar $[14,18]$ and lower than the reports from southern [17] and south-west Ethiopia [16]. The specificity for the non-falciparum species in the current study was comparable to the reports of some studies [14] higher in some others [18] and lower in elsewhere [16, 17]. The differences in the specificity of the RDT could be due to the aforementioned reasons [19].

The RDT had high NPV, meaning that it was reliable in ruling out malaria. Similarly, the higher PPV means that patients will be correctly diagnosed as positive for malaria and avoids unnecessary treatment.

The overall prevalence of malaria in the study area was very high, as detected by either the SD BIOLINE malaria $\mathrm{Ag}$ P.f/P.v (42.3\%) or the smear microscopy (40.5\%). The result was higher than the report from Oromia Regional State in Ethiopia (23.2\%) [14] While it was in agreement with a report from three regions in Ethiopia [20]. The high prevalence could be partly explained by the fact that the study was conducted in a peak malaria transmission season in the country. Also, the malaria transmission pattern in Ethiopia is highly seasonal and unstable. Because of this unstable transmission and infrequent exposure to infection, immunity is generally under-developed and all age groups are at risk of malarial disease [19] On the other hand, it might be due to development of anti-malarial or insecticide resistance in the area [21]. The knowledge, attitude and practice of the participants could also be a factor [19]. However, these assumptions should be evaluated with further studies. The high prevalence of malaria, despite the tremendous effort to distribute bed nets and apply outdoor insecticides, heralds the need to evaluate the malaria control system in the area and beyond.

Current subjective or objective fever (axillary temperature of $>37.5^{\circ} \mathrm{C}$ ) was the most common presenting symptom by the participants (table 5). Fever detects only 88.3\% malaria. This could be explained by the fact that individuals may carry parasites without symptoms. On the other hand, the significant overlap of malaria symptoms with other tropical diseases might have impaired the specificity of fever and encouraged the indiscriminate use of anti-malarials for managing febrile conditions in endemic areas. Studies of fever cases in Philippines, Sri Lanka, Thailand, Mali, Chad, Tanzania and Kenya have shown high percentages of malaria over-diagnosis when using fever as a clinical diagnostic too $[22,23]$. Comprehensive investigation to identify the etiologic agents of febrile illnesses could be helpful in the study area and beyond. Defining the malaria-attributable fraction to estimate the frequency of true febrile malaria among all febrile cases, by fitting the risk of fever as a function of parasite density using a logistic regression model, would be of paramount importance [24].

Some of the interference of SD malaria Ag Pf/Pv POCT kit 
with relevant interfering specimens such as hemolytic rheumatoid factors contained samples and lipemic, ictrec samples with investigated.

\section{Conclusions and Recommendations}

The SD malaria Ag Pf/Pv POCT RDT test showed good sensitivity and specificity with an excellent agreement to the reference smear microscopy. The RDT could therefore be used in place of smear microscopy, which in poor set-ups cannot be used microscopy routinely at health post.

Even though SD malaria $\mathrm{Ag} \mathrm{Pf} / \mathrm{Pv}$ POCT RDT test is used instead of microscopy by health extension workers, it is useful to put malaria RDT quality control system in place for health systems operating to monitor technical skill of the health extension worker. SD malaria Ag Pf/Pv POCT RDT could be used for epidemiological studies and the results the same as smear microscopic. Future research targeted to RDT evaluation should consider the use of a more sensitive reference standard such as PCR.

\section{Abbreviations}

$\begin{array}{ll}\text { AAU } & \text { Addis Ababa University } \\ \text { AFENET } & \text { African Field Epidemiology Network } \\ \text { CDC } & \text { Centers for Disease Control and Prevention } \\ \text { DHD } & \text { District Health Office } \\ \text { FMOH } & \text { Federal Ministry of Health } \\ \text { HRP2 } & \text { Histidine reaches protein II } \\ \text { ITNs } & \text { Insecticide treated net } \\ \text { NPV } & \text { Negative predictive value } \\ \text { PLDH } & \text { Plasmodium Lactose Dehydrogenase } \\ \text { PPV } & \text { Positive predictive value } \\ \text { RDT } & \text { Rapid Diagnostic test } \\ \text { WHO } & \text { World health organization } \\ \text { ZHD } & \text { Zonal Health Department }\end{array}$

\section{Acknowledgements}

We would like to acknowledge AFENET /CDC Mini GRANTS PROGRAM for the fully covering of financial support to conducting this assessment.

We also would like to acknowledge East Shewa Zone Health Department, Adama District Health Office and health facilities for facilitating us throughout whole research process.

Last, but not least, we would like to acknowledge study participants for their voluntarily participated in this study.

\section{References}

[1] Laura Penny, Paul Neuwald, and Mickey Urdea. Biomarkers for infectious Disease Diagnostics in the Developing World: Rapid Home based Diagnosis of Malaria in Symptomatic Individuals and Screening of Asymptomatic Pregnant Women, July 2006.
[2] Baboo K. S, N dayambaje I. et. a. Effectiveness of Rapid diagnostic test for malaria diagnosis in children 15 years of age of Nchelenge District in the Luapula province. medical journal of Zambia, Volume 35, Number 4 (2008) page 160.

[3] Qualitative Study on Malaria Prevention and Control in Oromia and Amhara Regional States in Ethiopia, Report Submitted to Academy for Educational Development (AED) by Addis Continental Institute of Public Health (ACIPH) 17 July, 2009.

[4] Manual of standard operating procedures for Assessment of Malaria Rapid Diagnostic Tests within the Product Testing Programme of the World Health Organization At US Centre's for Disease Control and Prevention, Atlanta Version Three 2010 For internal use.

[5] The American Journal of Tropical Medicine and Hygienewww.ajtmh.org 1. Am J Trop Med Hyg December 2007 vol. 77 no. 6 Suppl 119-127.

[6] Clinical Microbiology Rev. 2008 January; 21 (1): 97-110. doi: 10.1128/CMR.00035-07 http://dx.crossref.org/10.1128\%2FCMR.00035-07 PMCID: PMC2223842.

[7] Clinical Microbiology Rev. 2005 January; 20 (1): 87-120. doi: 10.1128/CMR.00024-03 http://dx.crossref.org/10.1128\%1FCMR.00024-03.

[8] Malaria in www.EthioDemographyAndHealth.Org.AynalemAdugna, accessed on 25/12/2011.

[9] Clinical Microbiology Reviewscmr.asm.org doi: 10.1128/CMR.15.1.66-78.2002 Clin. Microbiol. Rev. January 2002 vol. 15 no. 1 66-78.

[10] Rapid diagnostic test for diagnosis of uncomplicated P.falciparum malaria, validation and ease of use of three new pan pLDH tests, final report Jan 2006.

[11] Bendezu et al., Field evaluation of a rapid diagnostic test (Parascreen ${ }^{\mathrm{TM}}$ ) for malaria diagnosis in the Peruvian Amazon Malaria Journal 2010, 9: 154.

[12] Beyene M, Bemnet A, et al. Comparison of CareStart ${ }^{\mathrm{TM}}$ HRP2/pLDH COMBO Rapid Malaria Test with Light Microscopy in North-west Ethiopia, Malaria Journal, 2012.

[13] Adugna W, Wakgari D, et al., Evaluation of CareStart ${ }^{\mathrm{TM}}$ malaria $\mathrm{Pf} / \mathrm{Pv}$ combo test for Plasmodium falciparum and Plasmodium vivax malaria diagnosis in Butajira area, southcentral Ethiopia, Malaria Journal, 2013.

[14] Alemu A, Abebe G, Tsegaye W, Golassa L: Climatic variables and malaria transmission dynamics in Jimma Town, South West Ethiopia. Parasit ectors 2011, 4:30. PubMed Abstract | BioMed Central Full Text PubMed Central Full Text.

[15] Gemeda A, Wakgari D, Ahmed A, Lindtjørn B: Shifting to Plasmodium vivax dominance at highlands of Ethiopia following the countrywide intensive use of artemetherlumefantrine throughout endemic areas since 2005: Can we neglect the role of climate and relapse cases?. Addis Ababa, Ethiopia: 13th World Congress on Public Health; April 23-27; 2012.

[16] Bell D, Wongsrichanalai C, Barnwell J: Ensuring quality and access for malaria diagnosis: how can it be achieved? Nature 2006, 4: S7-S20. 
[17] Endeshaw T, Gebre T, Ngondi J, Graves PM, Shargie EB, Ejigsemahu Y, Ayele B, Yohannes G, Teferi G, Messele A, Zerihun M, Genet A, Mosher AW, Emerson PM, Richards FO: Evaluation of light microscopy and rapid diagnostic test for the detection of malaria under operational field conditions: a household survey in Ethiopia. Malar J 2008, 7:118. PubMed Abstract | BioMed Central Full Text | PubMed Central Full Text.

[18] Lemma H, Byass P, Desta A, Bosman A, Costanzo G, Toma L, Fottrell E, Marrast AC, Ambachew Y, Getachew A, Mulure N, Morrone A, Bianchi A, Barnabas GA: Deploying artemetherlumefantrine with rapid testing in Ethiopian communities: impact on malaria morbidity, mortality and healthcare resources. Trop Med Int Health 2009, 15: 241-250. PubMed Abstract | Publisher Full Text.

[19] WHO: The use of malaria diagnostic tests. Geneva, Switzerland: World Health Organization; 2006.

[20] WHO: Malaria rapid diagnostic test performance: results of WHO product testing of malaria RDTs: Rounds 1 and 2 (2008-2009). Geneva: World Health Organization; 2010.
[21] Negash K, Kebede A, Medhin A, Argaw D, Babaniyi O, Guintran JO, Delacollette C: Malaria epidemics in the highlands of Ethiopia. East African Med J 2005, 82: 186-192. PubMed Abstract.

[22] Ashton R, Kefyalew T, Tesfaye G, Counihan H, Yadeta D, Cundill B, Reithinger R, Kolaczinski JH: Performance of three multi-species rapid diagnostic tests for diagnosis of Plasmodium falciparum and Plasmodium vivax malaria in Oromia Regional State. Ethiopia. Malar J 2010, 9: 297. BioMed Central Full Text.

[23] Chanie M, Erko B, Animut A, Legesse M: Performance of CareStart ${ }^{\mathrm{TM}}$ Malaria $\mathrm{Pf} / \mathrm{Pv}$ Combo tests for the diagnosis of Plasmodium falciparum and Plasmodium vivaxinfections in Afar Region in North East Ethiopia. Ethiop J Health Dev 2011, 25: 206-211.

[24] Okell L, Ghani AC, Lyons E, Drakeley CJ: Submicroscopic infection in Plasmodium falciparum-endemic populations: a systematic review and meta-analysis. J Infect Dis 2009, 200: 1509-1517. PubMed Abstract | Publisher Full Text. 